\title{
Computation of 3D Water Flows by the Double Potential Method for the Simulation of Electromagnetic Water Purification
}

\author{
Nikita Tarasov ${ }^{1, \star}$, Sergey Polyakov ${ }^{1,2, \star \star}$, and Tatiana Kudryashova ${ }^{1, \star \star \star}$ \\ ${ }^{1}$ Keldysh Institute of Applied Mathematics of RAS, 4 Miusskaya square, 125047 Moscow, Russia \\ ${ }^{2}$ National Research Nuclear University MEPhl, 31, Kashirskoe shosse, 115409, Moscow, Russia
}

\begin{abstract}
In this paper, we discuss the modeling of the electromagnetic water purification. This model requires a velocity distribution in a study domain. For that purpose, the double potential method for simulating incompressible viscous fluid flows was used. The system of equations was discretized with the help of the finite volume method using an exponential transformation for the vortex calculation. As a result, a software implementation of the developed numerical scheme was obtained. The simulation of the three-dimensional flow was carried out in a study domain. The results were compared with Ansys CFD. The comparison showed a good degree of consistency between the two distributions. Using the obtained velocity field, we simulated the process of water purification using the induction of the electromagnetic field.
\end{abstract}

\section{Introduction}

The investigation of three-dimensional fluid flow modeling in this paper stems from the problem of the electromagnetic purification of fluid from the iron ion impurity. For that purpose, the equations of convection-diffusion with the addition of the Lorentz force are used [1]. This system of equations requires a velocity field distribution for the simulation.

Previously, we used the Navier-Stokes equations in the variables of the stream function - the vortex [1], when simulating this process in a two-dimensional formulation. This formulation is inapplicable in the case of modeling three-dimensional flow. Setting in the natural variables (velocity - pressure) has several drawbacks, including the complexity of the resulting equation for calculating the pressure, as well as the substantial instability of the numerical solution when using the finite volume method with a cell-centered control volume [2]. Besides, there is a generalization of the stream function to the three-dimensional case called vector potential $[2,3]$. The main difficulty, in this case, is the formulation of boundary conditions.

To overcome the difficulties described, it is possible to use the double potential method developed in the papers [4-6]. The essence of this method is to represent the speed as the sum of the curl of the vector potential and the gradient of the scalar potential. This allows us, on the one hand, to exclude pressure from consideration (it can be calculated later using a known velocity field), on the other hand, to avoid a set of complex boundary conditions on the vector potential.

\footnotetext{
$\star$ e-mail: nikita_tarasow01@mail.ru

$\star \star$ e-mail: polyakov@imamod.ru

$\star \star \star$ e-mail: kudryashova@imamod.ru
} 


\section{Mathematical model and numerical method}

The main idea of the double potential method consists in representing the velocity as [4-6]:

$$
\vec{u}=\vec{\nabla} \times \vec{A}-\vec{\nabla} \phi
$$

where $\vec{u}$ is the velocity vector, $\vec{A}$ is the vector potential, $\phi$ is the scalar potential, $\vec{\nabla}$ is the nabla operator. Moreover, we need to define the vortex $\vec{\omega}$ as follows

$$
\vec{\omega}=\vec{\nabla} \times \vec{u} .
$$

Using the curl property $\vec{\nabla} \times(\vec{\nabla} \times \vec{A})=\vec{\nabla}(\vec{\nabla} \cdot \vec{A})-\Delta \vec{A}$ and demanding $\vec{\nabla} \cdot \vec{A}=0$ we can get the following equation to calculate the vector potential by substituting (1) in (2):

$$
\Delta \vec{A}=-\vec{\omega},
$$

where $\Delta$ is the Laplace operator. The equation of the scalar potential has the standard form

$$
\Delta \phi=0 .
$$

The vortex transfer equations, obtained from the Navier-Stokes equations, are, in unitless form

$$
\frac{\partial \vec{\omega}}{\partial t}+(\vec{u}, \vec{\nabla}) \vec{\omega}-(\vec{\omega}, \vec{\nabla}) \vec{u}=\frac{1}{\operatorname{Re}} \Delta \vec{\omega},
$$

where $\mathrm{Re}$ is the Reynolds number, and $(\cdot, \cdot)$ denotes the scalar product. The velocity equation (1) is solved with the following boundary conditions for the potentials:

$$
\overrightarrow{A_{\tau}}=\frac{\partial \overrightarrow{A_{n}}}{\partial \vec{n}}=0 \quad \text { on } \quad \delta \Omega, \quad \frac{\partial \phi}{\partial \vec{n}}=-(\vec{u}, \vec{n}) \quad \text { on } \quad \delta \Omega,
$$

where $\delta \Omega$ is the domain border, $\overrightarrow{A_{\tau}}$ is the tangential component of the vector potential, $\overrightarrow{A_{n}}$ is the normal component of the vector potential, $\vec{n}$ is the normal to the domain border.

To conclude, the system of equations for modeling the viscous incompressible fluid flow using the double potential method is given by (3) - (5). The boundary conditions for potentials are given by (6), the boundary conditions for the vortex are determined from (2) using the substitution of the known boundary velocity distribution.

We used the finite volume method [7] with the cell-centered control volume to construct a numerical scheme. The Jacobi iteration method was used to resolve the algebraic system obtained by the discretization of the equations (3) and (4). For the discretization of the equation (5), an exponential transformation [8] was used, then, the finite volume method was used by the centers of the mesh elements for the approximation of the spatial derivatives. The approximation of the time derivative was performed using an explicit scheme. This numerical algorithm is described in detail in [9].

\section{Simulation results}

The simulation of the cleaning of an incompressible viscous fluid from impurities was carried out for the geometry shown in the Figure 1. The numbers in the Figure 1 are unitless. This computational area has one inlet at the left and two outlets at the right. Contaminated fluid flows from left to right, with a wall separating the flow into two parts (Figure $1 \mathrm{left}$ ). The Poiseuille flow is defined at the inlet boundary, the soft boundary conditions at the outlet boundaries and the no-slip conditions $(\vec{u}=0)$ on 

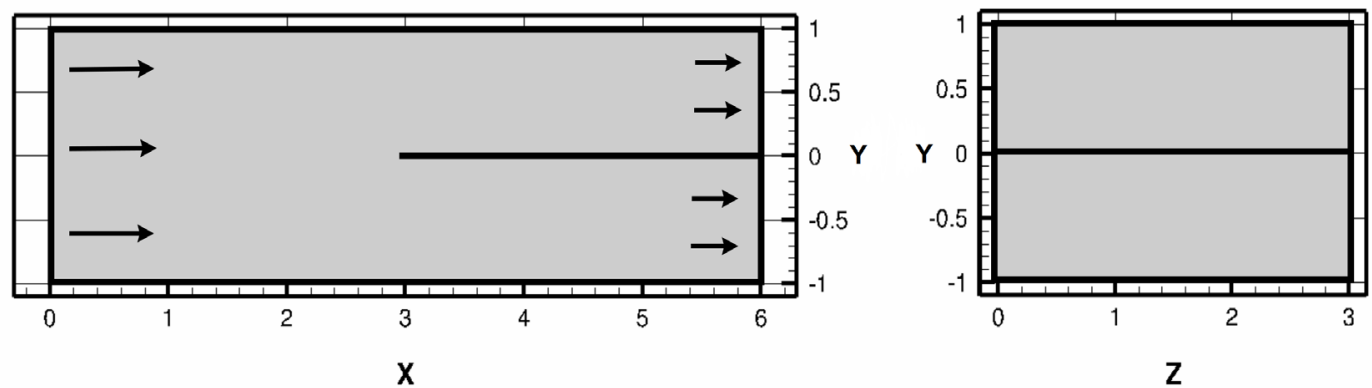

Figure 1. Domain geometry
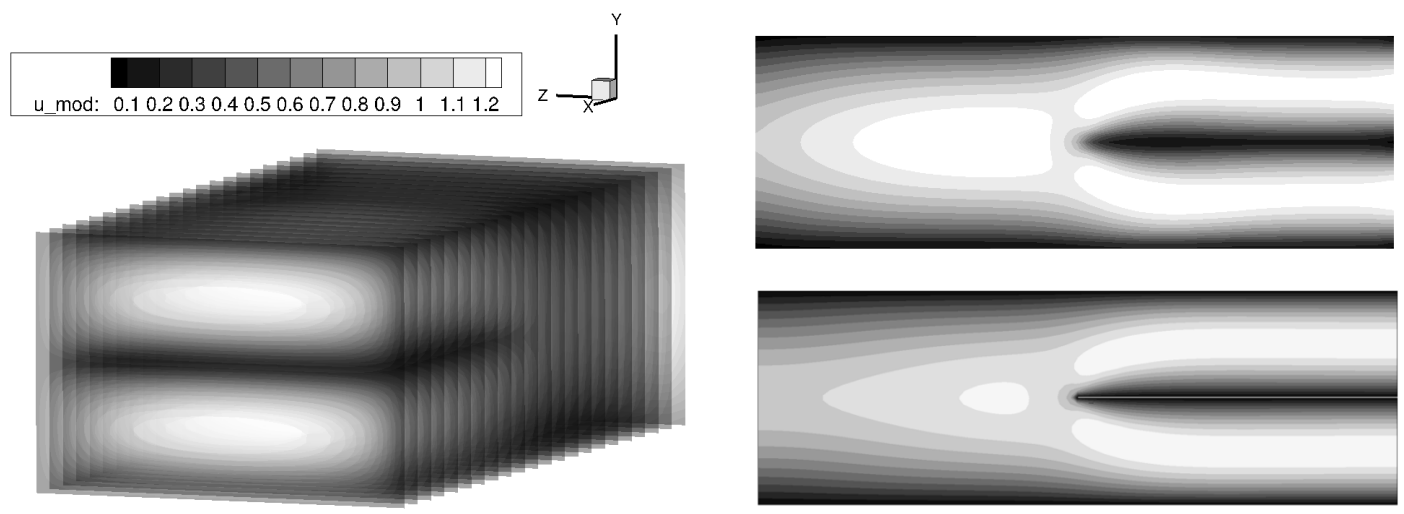

Figure 2. Distribution of the velocity modulus in the general view (left) and at the $Z=1.5$ plane, calculated by the double potential method (top right) and Ansys CFD (bottom right)

the walls are set. At the same time, part of the reservoir (from 0.0 to 2.9 in the $X$ direction) is under the influence of the induced electromagnetic field in the positive direction of the Z-axis. Due to this separation, the liquid saturated with impurities flows from the lower outlet. The purified liquid flows from the top one. This model describes the fluid flow purification process by electromagnetic field treatment.

The simulation was performed on a hexahedral mesh consisting of 170000 elements. The time step $\tau$ was taken equal to $9.868 \cdot 10^{-5}$ in dimensionless units. The Reynolds number was assumed to be 50, hence laminar flow. As a result, a static solution was obtained in 243100 iterations. The distribution of the velocity module is presented in the Figure 2. It was compared with the solution of a same problem obtained using the Ansys CFD Fluent software package [10]. The velocity distribution obtained by the double potential method is in good qualitative agreement with the results obtained by Ansys, which is shown by the behavior of the flow on the domain inlet and after the separator part.

The model described in [1] was used to calculate the distribution of the iron ions impurities concentration, which is well generalized to a three-dimensional case. Based on this model, a numerical method was obtained and the resulting algorithm was implemented in a computer code package, through which the simulation was carried out. The resulting static concentration distribution is presented in the Figure 3. The method of purification under investigation predicts a $40 \%$ purification with 

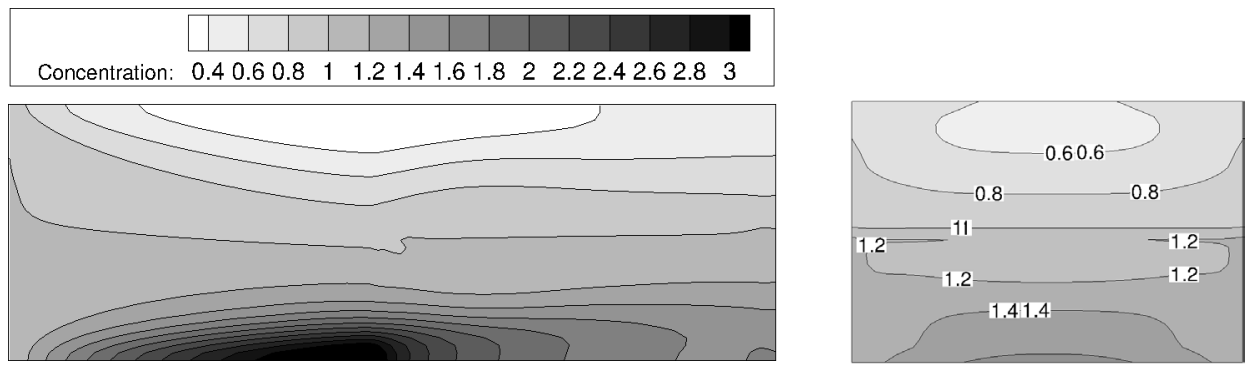

Figure 3. Distribution of the impurity concentration at the $Z=1.5$ plane (left) and at the $X=6$ plane (right)

one run of the fluid. This technique allows the cleaning quality improvement by assembling similar reservoirs in a cascade.

\section{Summary}

A numerical algorithm for simulation the flow of a viscous incompressible fluid in a three-dimensional case was developed and implemented in a code package. The distribution of the velocity field in the three-dimensional region was calculated. After that, these velocities were applied to simulate the electromagnetic purification of fluid flow from iron ions impurities. The distribution of the impurity concentration in the computational domain was obtained, which made it possible to estimate the resulting purification rate achieved with the electromagnetic treatment method.

\section{Acknowledgements}

The work was supported by Russian Fund for Basic Research, Projects No. 18-51-18004-bolg-a, 18-07-01292-a, 18-07-00841-a.

\section{References}

[1] S.V. Polyakov, Yu.N. Karamzin, T.A. Kudryashova, N.I. Tarasov, Mathematica Montisnigri XL, 110-126 (2017)

[2] P.J. Roache Computational Fluid Dynamics (Hermosa Publishers, New Mexico, 1976)

[3] C. Fletcher Computational Techniques for Fluid Dynamics 2 (Springer-Verlag, Berlin, Heidelberg, 1991)

[4] S.M. Richardson, Numerical solution of the three-dimensional Navier-Stokes equations. Doctoral dissertation (Department of Chemical Engineering and Chemical Technology, Imperial College of Science and Technology, London, 1976)

[5] S.M. Richardson, A.R. Cornish, Journal Fluid Mech. 82 part 2, 309-319 (1977)

[6] S.G. Gegg, A dual-potential formulation of the Navier-Stokes equations (Retrospective Theses and Dissertations, Iowa State University, 1987)

[7] R. Eymard, T.R. Gallouet, R. Herbin, Handbook of Numerical Analysis 7, 713-1020 (2000)

[8] S.V. Polyakov, Mathematica Montisnigri XXV, 1-16 (2012)

[9] T. Kudryashova, S. Polyakov, N. Tarasov, Computational Science - ICCS 2019, Lecture Notes in Computer Science 11539, 568-579 (2019)

[10] T. Stolarski, Y. Nakasone, S. Yoshimoto, Engineering Analysis with ANSYS Software (Butterworth-Heinemann, 2018) 